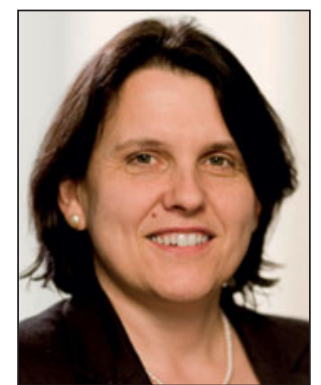

\section{Claudia Felser}

Guest Editor for this issue of MRS Bulletin

Max Planck Institute for Chemical Physics of

Solids, Germany; tel. +49-6131-392-1284;

and email felser@cpfs.mpg.de.

Felser is the director of the Max Planck Institute for Chemical Physics of Solids. She studied chemistry and physics at the University of Cologne. Her research interests include Heusler compounds, spintronics, superconductors, and topological insulators. She was the distinguished lecturer of the IEEE Magnetic Society. She received the SUR Grant from IBM and in 2011 received an ERC Advanced Grant. She is a Fellow of the Institute of Physics, London, and the American Physical Society.

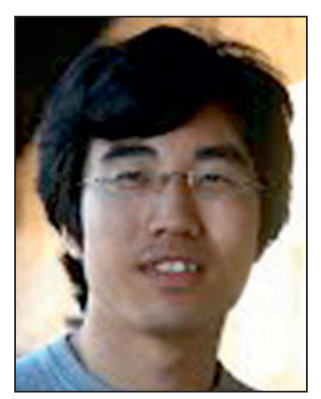

\section{Xiao-Liang Qi}

Guest Editor for this issue of MRS Bulletin

Stanford University, USA; tel. 650-724-5259;

and email xlqi@stanford.edu.

Qi is an assistant professor of physics at Stanford University. He received his bachelor's degree (2003) and PhD degree (2007) from Tsinghua University. He works in condensedmatter theory, and his main research interests include in topological states of matter, quantum entanglement, and holographic duality. He received the 2014 Sackler International Prize in Physics, the 2011 Hermann Kümmel Early Achievement Award in Many-Body Physics, the 2011 Packard Fellowship, and a 2010 Sloan Research Fellowship.

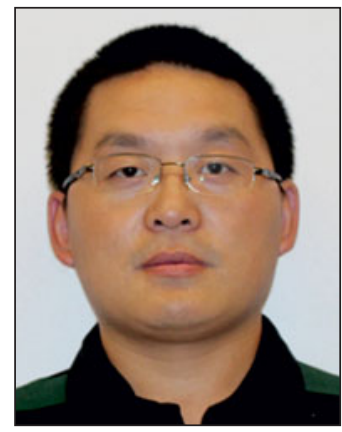

\section{Cui-Zu Chang}

Francis Bitter Magnet Laboratory,

Massachusetts Institute of Technology,

USA; email czchang@mit.edu.

Chang is a postdoctoral associate working with Jagadeesh Moodera at the Massachusetts Institute of Technology. He received his BSc degree in 2007 from Shandong University and PhD degree in 2013 from Tsinghua University. His thesis work involved searching for quantum anomalous Hall effect in quantum-well films of magnetically doped TI under the supervision of Qi-Kun Xue. $\mathrm{He}$ is actively involved in the topological insulator field.

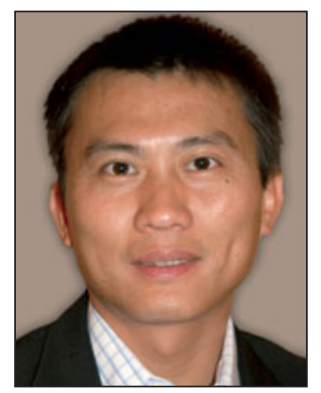

\section{Yi Cui}

Department of Materials Science and Engineering, Stanford Institute for Materials and Energy Sciences, SLAC National Accelerator Laboratory, USA; tel. 650-723-4613; and email yicui@stanford.edu.

Cui is the David Filo and Jerry Yang Faculty Scholar in the Department of Materials Science and Engineering at Stanford University with a joint appointment in the SLAC National Accelerator Laboratory. He received his BS degree in chemistry from the University of Science and Technology of China (1998) and his PhD degree in chemistry from Harvard University (2002). His research interests include nanomaterials for energy storage, solar cells, transparent electronics, topological insulators, environmental, and biotechnology. Honors include the Wilson Prize (2011), a Sloan Research Fellowship (2010), the KAUST Investigator Award (2008), the ONR Young Investigator Award (2008), the MDV Innovators Award (2007), and the Technology Review World Top Young Innovator Award (2004).

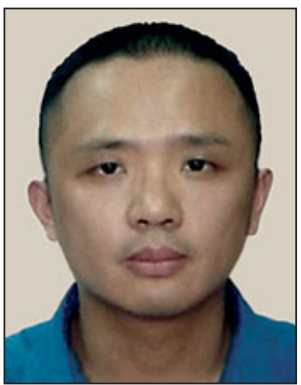

Xi Dai

Institute of Physics, Chinese Academy of

Sciences, China; email daix@aphy.iphy.ac.cn.

Dai is a professor at the Institute of Physics of the Chinese Academy of Sciences since 2006. He received his BS degree in 1993 from Zhejiang University and his PhD degree in 1999 from the Chinese Academy of Sciences. He was a postdoc at Hong University of Science and Technology, Boston College, and Rutgers University until 2004, and then worked as a research assistant professor at the University of Hong Kong until 2006. His main research interests are focused on the $a b$ initio studies of strongly correlated systems and topological materials.

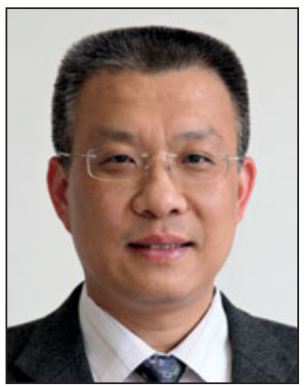

\section{Zhong Fang}

Institute of Physics, Chinese Academy of

Sciences, China; tel. 86-10-82648038;

and email zfang@aphy.iphy.ac.cn.

Fang is deputy director of the Chinese Academy of Sciences' Institute of Physics and has been a professor there since 2003. He received his PhD degree from Huazhong University of Science and Technology in 1996, and then visited Japan and the United States until 2003. His research focus is in computational condensed-matter physics, especially correlated electron systems and topological insulators. He has published more than 120 papers and is a Fellow of the American Physical Society.

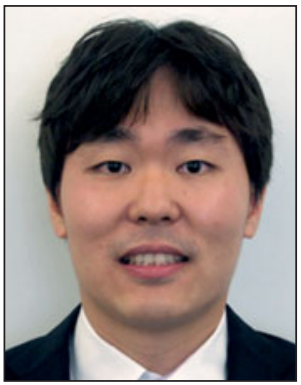

\section{Seung Sae Hong}

Department of Applied Physics, Stanford

University, USA; email seungsae@stanford.edu. Hong is currently a postdoctoral fellow in the group of Harold Y. Hwang at Stanford University. He received his BS degree in physics from Seoul National University in 2007 and completed his $\mathrm{PhD}$ degree in applied physics from Stanford University in 2013, supervised by Yi Cui. His research interests include physics and materials science of low-dimensional materials, with an emphasis on topological insulators and oxide interfaces.

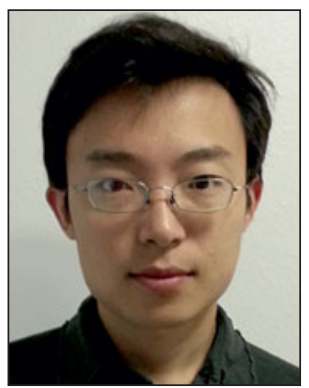

\section{Desheng Kong}

Department of Materials Science and

Engineering, Stanford University, USA; email desheng@stanford.edu.

Kong is a PhD student in the Department of Materials Science and Engineering at Stanford University supervised by Prof. Yi Cui. He received his BS degree in physics from Peking University in 2008. His graduate research focuses on the controlled synthesis of metal chalcogenide nanomaterials for applications in electronics and energy conversion. 


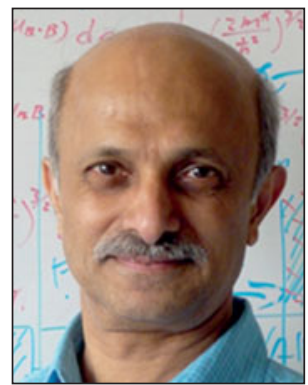

Jagadeesh Moodera

Francis Bitter Magnet Laboratory and Department of Physics, Massachusetts Institute of Technology, USA; tel. 617-253-5423; and email moodera@mit.edu.

Moodera is a senior research scientist and a group leader in the Physics Department at the Massachusetts Institute of Technology. He obtained a PhD degree in physics from the Indian Institute of Technology (IIT). His research interests include: spin tunneling, co-existence of ferromagnetism and superconductivity, superconductors in an internal exchange field, molecular spintronics, spin transport and exchange effects in topological insulator films, and Majorana fermions. He is a visiting professor at Eindhoven University of Technology, The Netherlands, and the University of Waterloo's Institute for Quantum Computing, Canada; and is a Distinguished Institute Professor at IIT (Chennai). He is a Fellow of APS and awards include the APS Oliver Buckley Prize and the NSF Competitiveness and Innovation Fellowship.

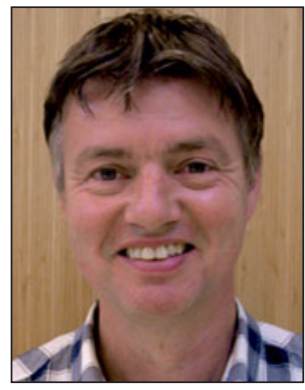

Anne de Visser

Van der Waals-Zeeman Institute, Faculty

of Science, University of Amsterdam,

The Netherlands; tel. +31205255732

and email: a.devisser@uva.n.

De Visser is an associate professor and principal investigator in the Quantum Electron Matter group in the University of Amsterdam's Physics Department. He obtained his PhD degree in physics in 1986 and was a postdoctoral researcher at the Low Temperature Research Center of the CNRS prior to his present position. His research interests include thermal, magnetic, and transport properties of strongly correlated electron systems, with a focus on the interplay of unconventional superconductivity and magnetism, as well as magnetotransport phenomena in high-magnetic fields of low-dimensional semiconductors and topological insulators.

\section{JAN IS}

\section{Cryogenic Systems}

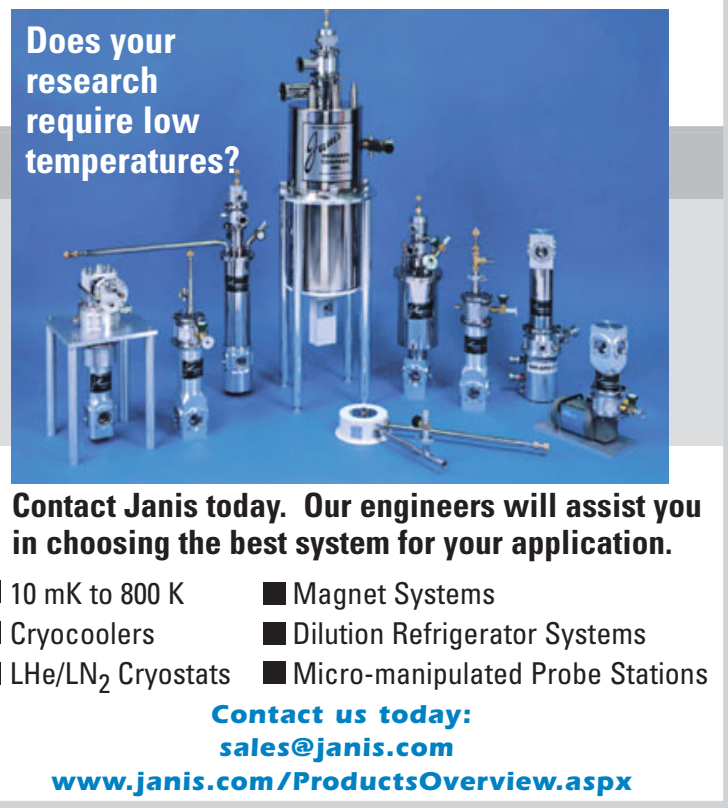

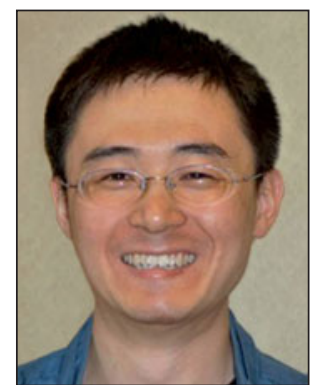

Peng Wei

Francis Bitter Magnet Laboratory,

Massachusetts Institute of Technology,

USA; email pwei@mit.edu.

Wei is a postdoctoral associate working with Jagadeesh Moodera at the Massachusetts Institute of Technology. He received his BSc degree from the University of Science and Technology of China (USTC) and his PhD degree from the University of California-Riverside, under the supervision of Jing Shi. His PhD thesis involved the study of thermoelectric properties of graphene and chemical potential tuning in exfoliated thin flake calcium doped $\mathrm{Bi}_{2} \mathrm{Se}_{3}$ nanodevices. His current focus is on $\mathrm{Tl}$ heterostructures, as well as novel systems hosting Majorana fermions.

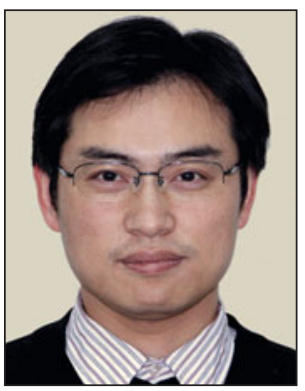

\section{Hongming Weng}

Institute of Physics, Chinese Academy of

Sciences, China; email hmweng@iphy.ac.cn.

Weng is an associate professor in the Chinese Academy of Sciences Institute of Physics. He received his BS (2000) and $\mathrm{PhD}(2005)$ degrees from Nanjing University. He then worked as a postdoc in Tohoku University's Institute for Materials Research until 2007, and then as an assistant professor of the Japan Advanced Institute of Science and Technology until 2010. His interests include developing the methods and programs for first-principles calculations and employing them to study quantum phenomena in condensed-matter physics. His recent research focuses on topological quantum states.

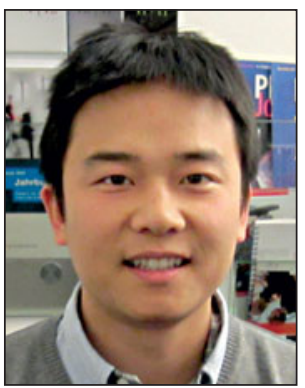

Binghai Yan

Max Planck Institute for Chemical Physics of Solids, Germany; tel. +49 (0)351 4646-2237; and email binghai.yan@cpfs.mpg.de.

Yan is a research group leader with a W2 professorship position at the Max Planck Institute for Chemical Physics of Solids. He received his $\mathrm{PhD}$ degree in physics from Tsinghua University, China, in 2008. His current research focuses on the theoretical study of topological insulators and oxide materials.

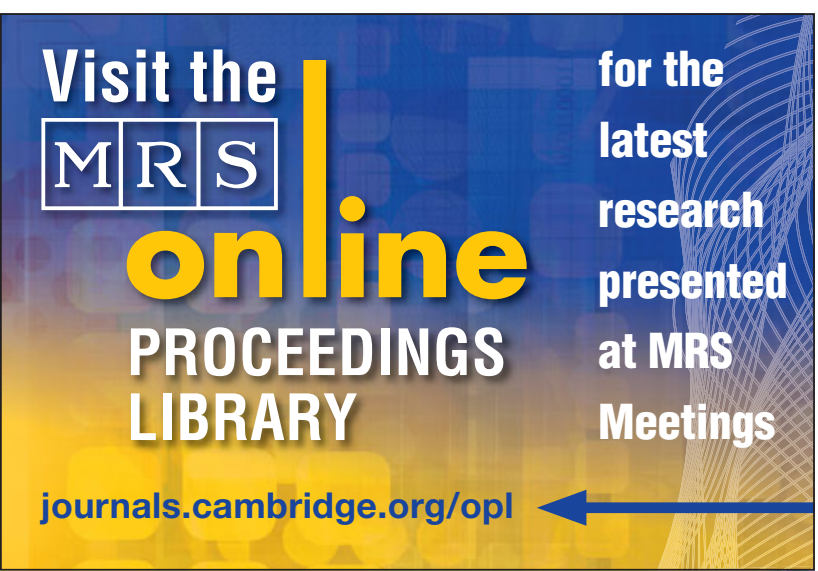

